\title{
Ischaemia of the medullary cone after stent-graft implantation in a patient with abdominal aortic aneurysm - a case study
}

\author{
Mariusz Wachowski ${ }^{1}$, Michał Polguj ${ }^{2}$, Janusz Ścibór ${ }^{3}$, Agata Majos $^{1}$ \\ ${ }^{1}$ Department of Radiological and Isotopic Diagnosis and Therapy, Medical University of Lodz, Lodz, Poland \\ ${ }^{2}$ Department of Angiology, Chair of Anatomy, Medical University of Lodz, Lodz, Poland \\ ${ }^{3}$ Department of Radiology and Diagnostic Imaging, M. Pirogow Hospital, Lodz, Poland
}

Videosurgery Miniinv 2018; 13 (1): 116-121

DOI: https://doi.org/10.5114/wiitm.2017.70326

\begin{abstract}
Preoperative visualization of the Adamkiewicz artery - the vessel which is to a great extent responsible for supplying blood to the medullary cone - is an important step which must be taken before initiating restorative procedures in the aorta. We present a case of a 67-year-old patient who underwent an intravascular stent-graft implantation procedure, due to clinical signs of abdominal aortic aneurysm. Routine pre-operative computed tomography examination failed to demonstrate the Adamkiewicz artery. On the second day after the surgery, as a result of unexpected clinical deterioration, an magnetic resonance imaging examination of the lumbar spine was carried out. Based on the magnetic resonance imaging images and clinical manifestations the diagnosis of ischaemia of the medullary cone was made. In our work we also present a deep analysis of the anatomy of small-sized vessels supplying blood to the spinal cord and discuss effective techniques which enable visualization of the Adamkiewicz artery.
\end{abstract}

Key words: aortic aneurysm, Adamkiewicz artery, spinal cord vascularization, medullary cone.

\section{Introduction}

Ischaemia of the medullary cone in comparison to ischaemia of the brain is a rare clinical occurrence. It is estimated that it constitutes only $1-2 \%$ of all neurological vascular pathologies [1]. All segments of the spinal cord can be affected by ischaemia but ischaemia at the medullary cone level is considered highly unusual [2]. In children, traumas and vascular malformations are the most common causes of ischaemia. In adult populations, predisposing factors of the disease include: aortic aneurysms (both of the thoracic and abdominal parts), venous thromboembolism, coagulopathies and all surgical procedures performed on the aorta - open repair surgery and endovascular methods. Additional factors which favour ischaemia of the medullary cone include ath- erosclerosis, arterial hypertension, diabetes and cardiac diseases [1, 2]. The disease is an important and difficult diagnostic problem due to the atypical clinical manifestation and various dynamics of symptoms, from unusual pain in the epigastric region to a dramatic course, including paraplegia and paresis. In more than $50 \%$ of cases, symptoms intensify within the first $12 \mathrm{~h}$ [1]. The most typical clinical manifestations of ischaemia at the medullary cone level include pain in the back area, radiating to lower extremities, dysuria caused by atonia of the bladder and anal sphincter dysfunction [2]. Other clinical symptoms are impaired sensation in the gluteal area and impaired sexual function in males.

The article will present a case of ischaemia of the medullary cone, as a complication of endovascular bifurcated stent graft implantation in a patient with 
abdominal aortic aneurysm. Although the long-term results of this method are still being evaluated, the use of the endovascular method in selected groups of patients is associated with superior perioperative outcomes in comparison to open repair procedures [3, 4].

\section{Case report}

A man, aged 67, was admitted to the Department of General and Vascular Surgery for an elective surgical procedure, due to clinical signs of abdominal aortic aneurysm, manifesting with periodical pain of changing intensity in the epigastric region (Photo 1 ). The aneurysm had been diagnosed 3 months prior to the patient's admission to the department. Before, the patient had suffered from episodes of stroke and undergone a bilateral trepanation of the skull, performed due to subacute subdural haematomas. His history also revealed long-lasting nicotine smoking and pharmacologically treated arterial hypertension. On admission, the patient was conscious and normally responsive. In a neurological examination, the patient was diagnosed with right hemiparesis, a consequence of previous strokes and neurosurgical procedures. In the epigastric region a mass, painless on palpation, resembling an aneurysm was revealed in a physical examination.

The patient was ordered an endovascular stent graft implantation procedure due to his clinical condition as well as due to the lower perioperative mortality and morbidity of this method in selected groups of patients in comparison to open surgery [3]. The approach to the abdominal aortic aneurysm was achieved by incising both the femoral arteries, through which a Zenith bifurcated stent graft was inserted. Systemic anticoagulation with $2500 \mathrm{IU}$ of unfractionated heparin (UFH) was accomplished before catheter insertion and manipulation into the femoral arteries.

Branches of an endovascular prosthesis were placed in the common iliac arteries. The stent graft body was placed in the abdominal aorta, below the place where the renal arteries arise, which allowed the aneurysm to be excluded from the circulation. A follow-up intra-operative angiography confirmed the proper placement and expansion of elements of the prosthesis (Photo 2). It also revealed proper contrasting of renal arteries, iliac external and internal arteries and no traces of leak.

On the first day following the stent graft implantation, the patient's condition dramatically deteri-

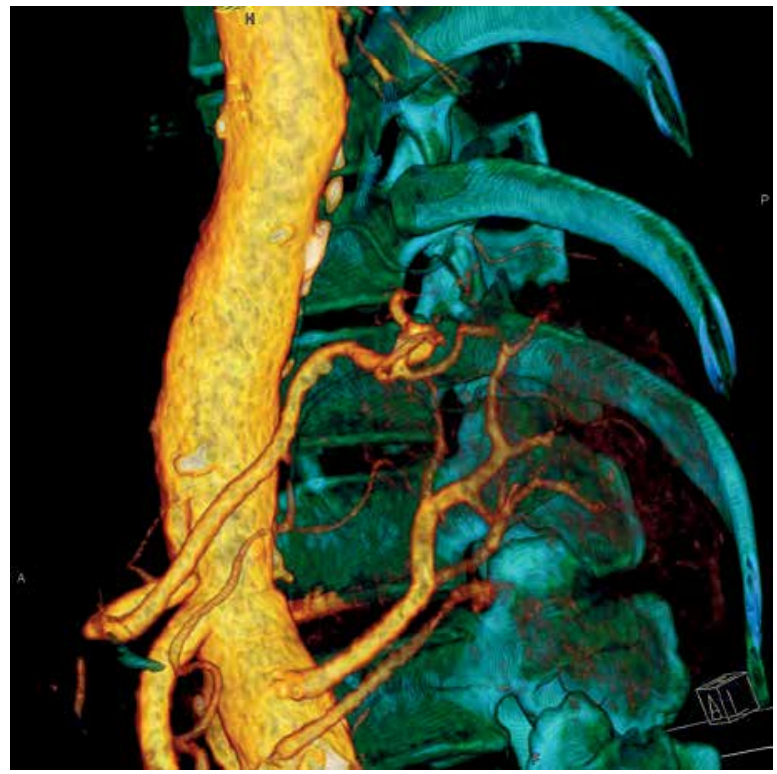

Photo 1. Pre-operative $C T$ examination, $3 \mathrm{D}$ reconstruction failed to demonstrate the Adamkiewicz artery

orated. He reported severe headaches, nausea and vomiting. He was periodically unresponsive and the verbal contact was impaired. The blood pressure was 200/90 mm Hg. Due to clinical symptoms and suspicion of another cerebral stroke, a single-phase computed tomography (CT) of the head was performed. The result of the examination allowed ischaemia to be excluded.

On the second day after the procedure, decreased muscle tone and decreased temperature in the lower extremities were observed. A neurological examination revealed bilateral flaccid paralysis as well as deep and superficial sensory disturbances of the lower limbs.

An magnetic resonance imaging ( $\mathrm{MRI}$ ) examination of the lumbosacral segment of the spine was performed urgently. It showed dilation of the whole distal segment of the spinal cord from the Th11 level to the mid L1 level, i.e. including its cone. Changes of signal intensity in T2-weighted images in the whole area of the cord at those levels, including the white and gray matter, were observed. The MRI results implied oedematous and ischaemic lesions (Photo 3).

Results of imaging examinations and clinical manifestations were the basis for diagnosing a stroke of the distal segment of the spinal cord, supplied by the Adamkiewicz artery. After 11 days of hospitalization, the patient, demonstrating flac- 

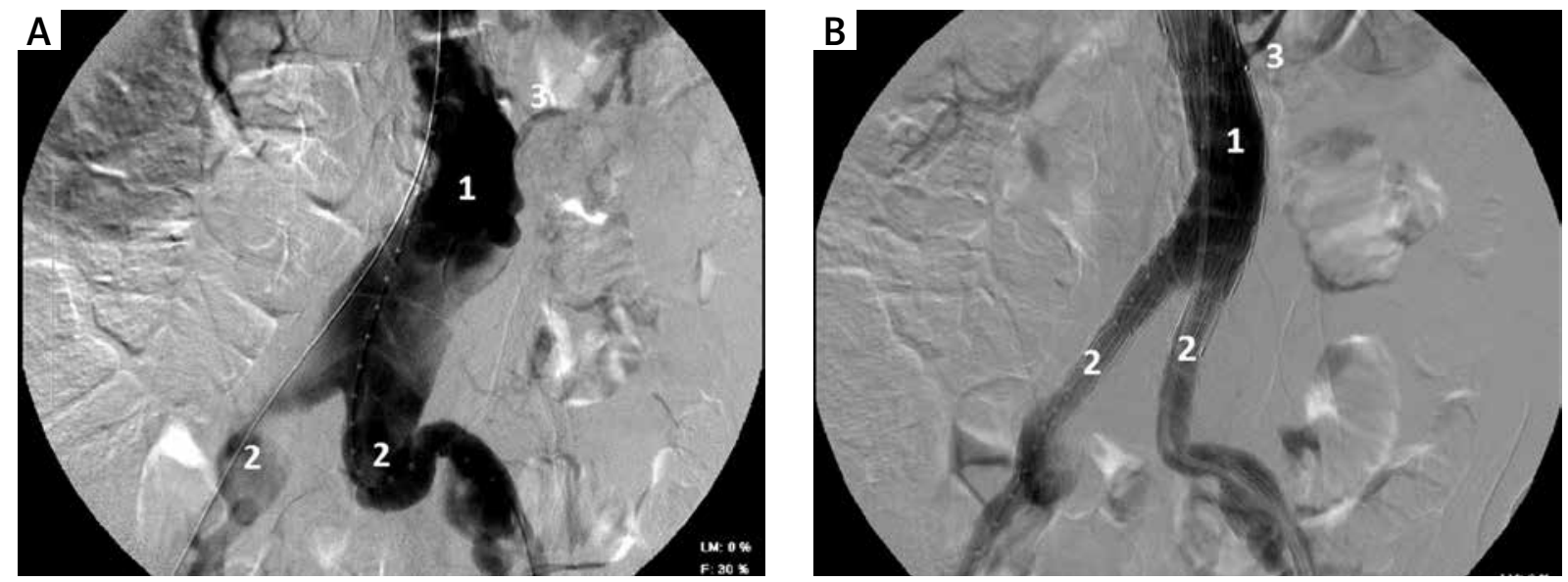

Photo 2. Routine DSA examination, L2-S1 level: A - abdominal aortic aneurysm-before intravascular stentgraft implantation, B - A follow-up intra-operative angiography; 1 - abdominal aorta, 2 - common iliac arteries, 3 - left renal artery

cid paralysis and sensory disturbances of the lower extremities, was moved to the Ward of Internal Medicine for rehabilitation treatment.

\section{Discussion}

The spinal cord, due to its length, is supplied with blood by a complex network of arterial vessels. Spinal arteries, i.e. branches of vertebral arteries, are the primary source of arterial blood. The arteries include anterior spinal arteries (ASAs) and posterior spinal arteries (PSAs). They are small-diameter vessels, running along the spinal cord. ASA and PSA diameters are in the range $0.2-0.8 \mathrm{~mm}$ and $0.1-0.4 \mathrm{~mm}$, respectively [5]. After a short distance, anterior spinal arteries (right and left) join and form one unpaired vessel, running downwards along the anterior median fissure of the spinal cord. It is estimated that the anterior spinal artery supplies blood to $2 / 3$ of the anterior portion of the spinal cord; it vascularizes the grey matter and the anterolateral part of the white matter. The pair of posterior spinal arteries run to the dorsal area of the medulla oblongata and next run downwards in the vertebral canal. The posterior spinal arteries vascularize $1 / 3$ of the posterior portion of the spinal cord, including the posterior funiculi [2]. In their course, the ASAs and PSAs join together with numerous vascular anastomoses and form the vasa corona, which supplies the spinal cord.
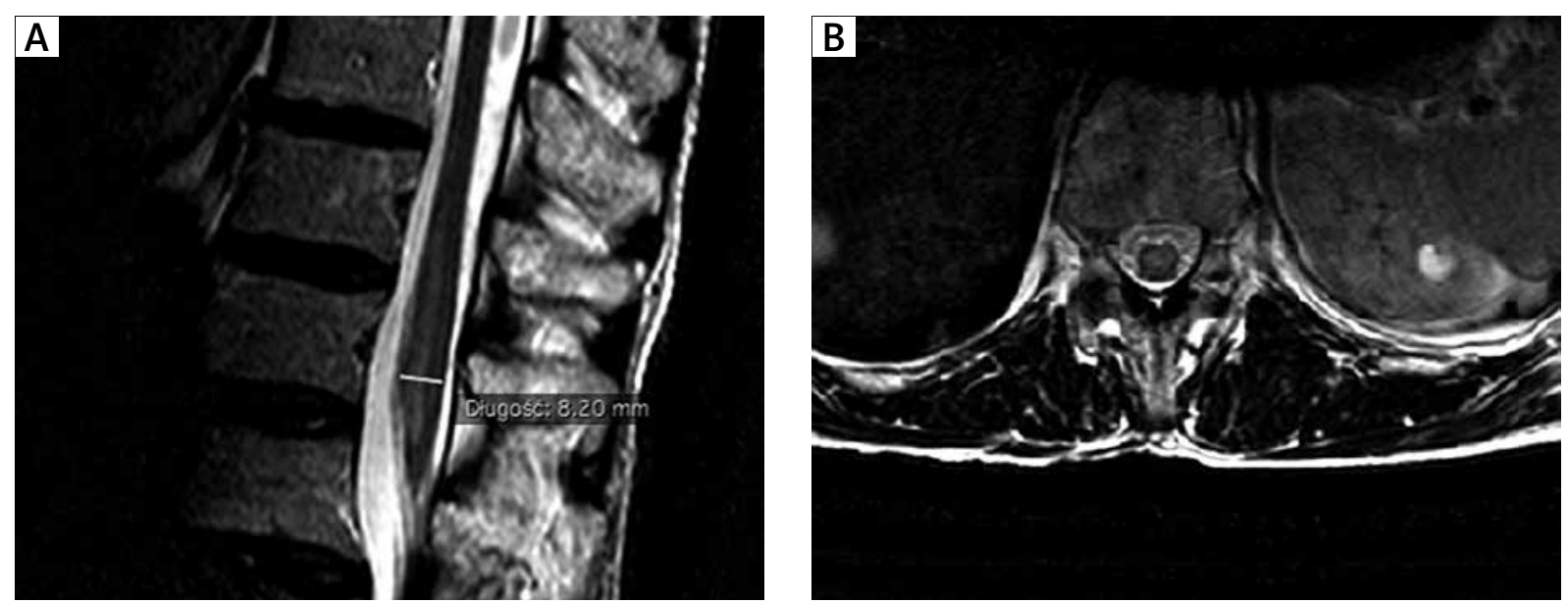

Photo 3. Magnetic resonance imaging examination, T2 weighted image. Dilatation and increased signal intensity of conus medullaris. A - Sagittal plane, B - Transverse plane 
Spinal branches which enter the vertebral canal through intervertebral and sacral foramina are another source of spinal cord vascularization. In the cervical segments they usually arise from the vertebral artery, the ascending cervical artery and deep cervical artery. In the thoracic part they usually arise from posterior intercostal arteries, in the lumbar segment from lumbar and iliolumbar arteries and, finally, in the sacral segment usually from lateral sacral arteries. Distal segments of spinal branches, i.e. anterior and posterior radicular arteries, play a key role in vascularization of the spinal cord. The Adamkiewicz artery, also called the great anterior radiculomedullary artery, is the largest anterior radicular artery, and the vessel which is to a great extent responsible for supplying blood to the medullary cone and the lumbar segments of the spine [6]. An extensive knowledge of topography and morphological variabilities of the Adamkiewicz artery is a key to understanding the pathophysiology and symptomatology of damage to the medullary cone as a result of its ischaemia.

In $68-73 \%$ of cases, the Adamkiewicz artery arises on the left side from the posterior branch of the intercostal artery (ICA) or the lumbar artery (LA), usually at the Th8-L1 level $(91 \%)[7,8]$. Next, in 80\% of cases it enters the spinal canal at the Th8-L4 level [9]. It is a small vessel, whose diameter ranges between 0.8 and $1.3 \mathrm{~mm}$ [7]. Koshino et al. in their study did not observe a significant correlation between the diameter of the Adamkiewicz artery and the diameter of ICA/LA, which the former vessel arises from [8]. Also, no correlation was found between the diameter of the great anterior radiculomedullary artery and sex or the body side along which the artery runs [10]. In some cases, it is possible to find more than one Adamkiewicz artery [11]. Twenty-four percent of patients included in a study conducted by Takase et al. had two Adamkiewicz arteries [12]. The Adamkiewicz artery consists of two parts: the ascending branch, which supplies the spinal cord as far as the inferior part of the thoracic segment, and the descending branch, which runs to the medullary cone. It joins the ASA and they together play an essential role in supplying blood to the spinal cord from the Th8 to the medullary cone.

Until recently, digital subtraction angiography (DSA) had been a basic method applied in imaging diagnostics of small-sized vessels. It enables one to visualize vessels due to administration of contrast agent through properly selected catheters. DSA is characterized by high spatial resolution and allows visualization of vessels $0.2 \mathrm{~mm}$ or above in diameter [13]. It is a reliable method which enables one to visualize the Adamkiewicz artery, see its course and identify the vessel it arises from. However, it should be remembered that DSA is an invasive technique, associated with possible adverse effects [12]. Moreover, the effectiveness of successful visualization of the Adamkiewicz artery with the application of this method ranges, according to various authors, from $43 \%$ to $65 \%$ [14]. According to a study conducted by Williams et al., factors which make this technique less diagnostically reliable include presence of parietal thrombi which close the lumen of the vessel, to which the contrast agent is administered, lack of possibility to insert a catheter into the true lumen of the dissecting aneurysm of the aorta, risk of toxic damage to the spinal cord by the contrast agent and thrombotic complications [15].

Hence, researchers started to seek more effective and safer techniques of visualizing small-sized vessels supplying blood to the spinal cord. Two non-invasive techniques, i.e. CT angiography and MRI angiography, were believed to be highly effective. In their study, Yoshioka et al. found that the accuracy in localizing the great anterior radiculomedullary artery was $80 \%$ for CT angiography and $66.7 \%$ for MRI angiography [7]. Other authors confirmed the advantage of CT angiography in visualizing the Adamkiewicz artery, particularly when the contrast agent is injected directly into the aorta (intra-aortic computed tomography angiography, IA-CTA). Nojiri et al. found that IA-CTA enabled the Adamkiewicz artery to be localized in all 27 patients who underwent this procedure. In $90 \%$ of them it was possible to identify the level from which the artery arose [14]. The contrast agent was administered through a catheter inserted into the right femoral artery or the left brachial artery, and its end was at the descending part of the thoracic aorta, just below the place where the left subclavian artery arises.

Also Uotani et al. observed in their study a greater diagnostic value of intra-arterial CT angiography in comparison to a technique involving intravenous administration of a contrast agent for the purpose of localizing the Adamkiewicz artery. The effectiveness of localization of the Adamkiewicz artery with the application of IA-CTA was 94.1\%, whereas it was only $60 \%$ in a group of patients who underwent in- 
tra-venous computed tomography angiography (IVCTA). It was possible to visualize the continuity of vessels from the aorta as far as the Adamkiewicz artery in $87.5 \%$ patients who underwent the IA-CTA procedure and in $55.6 \%$ of patients who underwent the IV-CTA procedure [16].

An interesting modification of the IV-CTA procedure was presented in a study conducted by Amako et al. [17]. The contrast agent was administered twice with a vascular catheter, directly inserted into the right atrium. The authors conducted CT scans after each injection - $20 \mathrm{~s}$ after the first injection and $35 \mathrm{~s}$ after the second injection. In the authors' opinion, such a method of application of a contrast agent might reduce its dilution in the pulmonary circulation and, in this way, improve visualization of the great anterior radiculomedullary artery, particularly in patients with circulatory insufficiency and in patients with aortic aneurysms. It was possible to identify the location of the Adamkiewicz artery in all 110 studied patients, and in $90 \%$ of cases this technique allowed the continuity of vessels to be traced from the aorta to the anterior spinal artery. Although the presented procedure shows high accuracy, we should bear in mind its possible restrictions. The patient is exposed to a double dose of X-ray radiation and administered a huge volume of a contrast agent, which might be a contraindication for patients with renal failure [17].

Another effective imaging technique which enables one to visualize the Adamkiewicz artery is contrast-enhanced MR angiography, performed with a 3.0-T imager. Its effective characteristics were confirmed in two independent studies, conducted by Bley and Takagi $[18,19]$. The effectiveness of visualization of the Adamkiewicz artery performed with a 3.0-T imager in the mentioned studies was $88 \%$ and $89.8 \%$ respectively. Due to increasing availability of such scanners and the non-invasive character of the examination, this technique might soon effectively replace the CT angiography procedure, in which a contrast agent is directly administered through catheters to the aorta or right atrium.

\section{Conclusions}

Visualization of the Adamkiewicz artery and identification of its morphology are important steps which must be taken before initiating restorative procedures in the aorta. They reduce the incidence of serious neurological complications, e.g. paralysis, which might occur after open and endovascular restorative procedures of aneurysms of the aorta

Both $\mathrm{CT}$ and MRI angiography, particularly performed with the use of state-of-the-art 3T scanners, are alternative techniques for DSA. We believe that it is worth considering including these methods in a standard protocol of preoperative examinations in patients with aortic pathologies.

\section{Conflict of interest}

The authors declare no conflict of interest.

\section{References}

1. Vargas MI, Gariani J, Sztajzel R, et al. Spinal cord ischemia: practical imaging tips, pearls, and pitfalls. AJNR Am J Neuroradiol 2015; 36: 825-30.

2. Wong J, Dufton J, Mior SA. Spontaneous conus medullaris infarction in a 79 year-old female with cardiovascular risk factors: a case report. J Can Chiropr Assoc 2012; 56: 58-65.

3. Synowiec T, Warot M, Burchardt M, et al. All dangerous types of endoleaks after endovascular aneurysm repair in a single patient. Videosurgery Miniinv 2015; 10: 290-4.

4. Wojtaszek M, Wnuk E, Maciag R, et al. Improving the results of transarterial embolization of type 2 endoleaks with the embolic polymer Onyx. Videosurgery Miniinv 2016; 11: 259-67.

5. Melissano G, Civilini E, Bertoglio L, et al. Angio-CT imaging of the spinal cord vascularisation: a pictorial essay. Eur J Vasc Endovasc Surg 2010; 39: 436-40.

6. Andrews BT, Kwei U, Greco C, et al. Infarct of the conus medullaris simulating a spinal cord tumor: case report. Surg Neurol 1991; 35: 139-42.

7. Yoshioka K, Niinuma $H$, Ohira A, et al. MR angiography and $C T$ angiography of the artery of Adamkiewicz: noninvasive preoperative assessment of thoracoabdominal aortic aneurysm. Radiographics 2003; 23: 1215-25.

8. Koshino T, Murakami G, Morishita K, et al. Does the Adamkiewicz artery originate from the larger segmental arteries? J Thorac Cardiovasc Surg 1999; 117: 898-905.

9. Milen MT, Bloom DA, Culligan J, et al. Albert Adamkiewicz (1850-1921): his artery and its significance for the retroperitoneal surgeon. World J Urol 1999; 17: 168-70.

10. Boll DT, Bulow H, Blackham KA, et al. MDCT angiography of the spinal vasculature and the artery of Adamkiewicz. AJR Am J Roentgenol 2006; 187: 1054-60.

11. Melissano G, Bertoglio L, Civelli V, et al. Demonstration of the Adamkiewicz artery by multidetector computed tomography angiography analysed with the open-source software OsiriX. Eur J Vasc Endovasc Surg 2009; 37: 395-400.

12. Takase K, Sawamura Y, Igarashi K, et al. Demonstration of the artery of Adamkiewicz at multi-detector row helical CT. Radiology 2002; 223: 39-45.

13. Nijenhuis RJ, Mull M, Wilmink JT, et al. MR angiography of the great anterior radiculomedullary artery (Adamkiewicz artery) 
validated by digital subtraction angiography. AJNR Am J Neuroradiol 2006; 27: 1565-72.

14. Nojiri J, Matsumoto K, Kato A, et al. The Adamkiewicz artery: demonstration by intra-arterial computed tomographic angiography. Eur I Cardiothorac Surg 2007; 31: 249-55.

15. Williams GM, Perler BA, Burdick JF, et al. Angiographic localization of spinal cord blood supply and its relationship to postoperative paraplegia. Vasc Surg 1991; 13: 23-33.

16. Uotani K, Yamada N, Kono AK, et al. Preoperative visualization of the artery of Adamkiewicz by intra-arterial CT angiography. AJNR Am I Neuroradiol 2008; 29: 314-8.

17. Amako M, Yamamoto Y, Nakamura K, et al. Preoperative visualization of the artery of Adamkiewicz by dual-phase CT angiography in patients with aortic aneurysm. Kurume Med J 2011; 58: 117-25.

18. Bley TA, Duffek CC, François CJ, et al. Presurgical localization of the artery of Adamkiewicz with time-resolved 3.0-T MR angiography. Radiology 2010; 255: 873-81.

19. Takagi $\mathrm{H}, \mathrm{Ota} \mathrm{H}$, Natsuaki Y, et al. Identifying the Adamkiewicz artery using 3-T time-resolved magnetic resonance angiography: its role in addition to multidetector computed tomography angiography. Jpn J Radiol 2015; 33: 749-56.

Received: 26.06.2017, accepted: 2.09.2017. 